\title{
Prognostic Impact of Left Ventricular Mass Severity According to the Classification Proposed by the American Society of Echocardiography/ European Association of Echocardiography
}

\author{
Andrea Barbieri, MD, Francesca Bursi, MD, MSc, Francesca Mantovani, MD, Chiara Valenti, MD, \\ Michele Quaglia, MD, Elena Berti, MD, Massimiliano Marino, PhD, \\ and Maria Grazia Modena, MD, FESC, FACC, Modena and Bologna, Italy
}

\begin{abstract}
Background: The American Society of Echocardiography (ASE) and European Association of Echocardiography (EAE) recommend the use of quantitative estimation of left ventricular (LV) mass and defined partition values for mild, moderate, and severe hypertrophy. However, the prognostic implications associated with this categorization are unknown.
\end{abstract}

Methods: In this observational cohort study of unselected adults undergoing echocardiography for any indication, LV hypertrophy was assessed using the ASE/EAE-recommended formula and measurement convention from LV linear dimensions indexed to body surface area. Mortality and incident hospitalizations for cardiovascular disease were the outcomes of this study.

Results: Of 2,545 subjects (mean age, $61.9 \pm 15.8$ years; $56.3 \%$ women), $52.9 \%$ had normal LV mass, and $15.4 \%$ had mild, $12.1 \%$ moderate, and $19.6 \%$ severe LV hypertrophy. During a mean follow-up period of $2.5 \pm 1.2$ years, 121 deaths and 292 incident hospitalizations for cardiovascular disease occurred. In multivariate models including age, gender, LV ejection fraction, wall motion score index, significant valvular disease, and atrial fibrillation, the adjusted hazard ratios for death were 1.81 (95\% confidence interval [Cl], 1.03-3.20; $P=.041)$ for mild, $2.31(95 \% \mathrm{Cl}, 1.33-4.01 ; P=.003)$ for moderate, and $2.30(95 \% \mathrm{Cl}, 1.39-3.79, P=.001)$ for severe LV hypertrophy. The adjusted hazard ratios for incident cardiovascular hospitalizations were 1.24 (95\% $\mathrm{Cl}, 0.84-1.82 ; P=.277)$ for mild, $2.02(95 \% \mathrm{Cl}, 1.42-2.88 ; P=.0001)$ for moderate, and $2.38(95 \% \mathrm{Cl}, 1.75-$ 3.22, $P<.0001$ ) for severe LV hypertrophy. After adjustment for known risk predictors, there was a 1.3-fold risk for death and cardiovascular disease events per category of LV mass $(P=.001)$.

Conclusions: In a cohort study of unselected adult outpatients, the categorization of LV mass according to the ASE/EAE recommendations offered prognostic information independently of age, gender, and other known predictors. (J Am Soc Echocardiogr 2011;24:1383-91.)

Keywords: Left ventricular hypertrophy, Left ventricular mass, Death, Echocardiography, Prognosis

Echocardiographically detected left ventricular (LV) hypertrophy has been shown to be a strong independent marker of cardiovascular risk both in the general population ${ }^{1-4}$ and in high-risk groups. ${ }^{5-9}$ Nonetheless, these studies have used a variety of indexation methods and partition values.

The American Society of Echocardiography (ASE) and European Association of Echocardiography (EAE) recommendations for

From the Department of Cardiology, Policlinico Hospital, Modena and Reggio Emilia University, Modena, Italy (A.B., F.B., F.M., C.V., M.Q., M.G.M.); and the Healthcare and Social Agency, Regione Emilia Romagna, Bologna Italy (E.B., M.M.).

Reprint requests: Francesca Bursi, MD, MSc, Department of Cardiology, Policlinico Hospital, Modena and Reggio Emilia University, Via del Pozzo 71, 41100 Modena, Italy (E-mail: bursi@libero.it).

0894-7317/\$36.00

Copyright 2011 by the American Society of Echocardiography.

doi:10.1016/j.echo.2011.08.012 chamber quantification ${ }^{10}$ conclude that LV mass from the ASE-recommended formula using LV linear dimensions indexed to body surface area (BSA) is preferred in the diagnosis of LV hypertrophy over linear measurements such as septal or posterior wall thickness. In addition, they propose sex-specific cutoff values to categorize the degree of LV hypertrophy as mild, moderate, and severe on the basis of the distribution of LV mass in relation to reference limits in an ethnically heterogeneous North American population. ${ }^{11}$

However, despite the widespread use of such descriptive terms in clinical practice, this categorization is, at present, inadequately substantiated by scientific data, underscoring the weaknesses inherent the use of arbitrary dichotomous threshold without providing insights into risks associated with different levels of LV mass. Therefore, aim of this study was to examine the prognostic implications of the ASE/ EAE partition values of LV mass in a large group of unselected outpatients referred to a tertiary care echocardiography laboratory. 
Abbreviations

BSA = Body surface area
$\mathbf{C I}=$ Confidence interval
$\mathbf{H R}=$ Hazard ratio
IVST = End-diastolic
interventricular septal
thickness

LV = Left ventricular

LVIDD = End-diastolic left ventricular internal dimension

PWT $=$ End-diastolic posterior wall thickness

RWT = Relative wall thickness

2D = Two-dimensional

WMSI $=$ Wall motion score

index

\section{Echocardiographic Data}

All exams were performed using an Acuson Sequoia ultrasound system (Siemens Medical Solutions USA, Inc., Mountain View, CA) and were performed and/or supervised by cardiologists fully trained in echocardiography with long-standing experience with the technique and intense hands-on training period with interpretation of $>750$ studies. $^{12}$

LV diameters as well as septal and posterior wall thickness were measured using the 2D-guided M-mode method in the short-axis view or the linear 2D method in the parasternal long-axis view. LV end-diastolic dimensions were measured at the onset of the QRS complex. LV ejection fraction was assessed using the biplane Simpson method or the Quinones method using LV end-systolic and end-diastolic diameters ${ }^{13}$ or visually estimated, a method that was documented to have accuracy comparable with that of the other methods in assessing LV ejection fraction. ${ }^{14}$

Regional LV systolic function was assessed using a standard 16-segment model. ${ }^{10}$ Segmental scores were assigned as follows: $1=$ normal or hyperkinesis, $2=$ hypokinesis, $3=$ akinesis, $4=$ dyskinesis, and $5=$ aneurysmal. The wall motion score index (WMSI) was derived as the sum of all scores divided by the number of segments visualized. Individual echocardiographic Doppler parameters (mitral inflow pattern, tissue Doppler, and Valsalva maneuver when necessary) were integrated to grade diastolic function in four stages: normal diastolic function; impaired relaxation with normal or near normal filling pressures (grade I/IV); impaired relaxation with moderate elevation of filling pressures, pseudonormal filling (grade II/IV); and impaired relaxation with marked elevation of filling pressures, restrictive filling (grades III-IV/IV), as previously described. ${ }^{15,16}$

Left atrial volume was assessed using the modified biplane Simpson method from apical four-chamber and two-chamber views and indexed to BSA. Measurements were obtained in end-systole from the frame preceding mitral valve opening. ${ }^{17,18}$

Each value represented the average of three consecutive beats.

Significant left-sided valve disease severity was defined as the presence of aortic or mitral prosthesis or the presence of greater than moderate native mitral or aortic valve stenosis or insufficiency, similarly to previous reports. ${ }^{19}$ Valve disease severity was defined according to the American Heart Association and American College of Cardiology guidelines for the management of valvular heart disease. ${ }^{20}$ Applying these guidelines, the cardiologist performing the exam graded valve disease as absent, mild, moderate, or severe, and this information was embedded in the echocardiographic report. The methods used included pulsed-wave and continuous-wave Doppler velocities and gradients, direct measurement of valve area planimetry, continuity equation, color Doppler to assess the jet width, or proximal isovelocity surface area for quantitative evaluation. The methods used to classify valve disease severity were at the discretion of the physician performing the exam, and often the final judgment was based on the combination of more than one method.

All measurements were performed online and entered in an electronic database at the time of the echocardiographic study. No modification from the original database was applied, and no measurement was made offline. Hence, the study consisted of a retrospective analysis of data entered in the electronic echocardiographic database.

\section{Mass}

LV mass was obtained using the ASE-recommended formula for the estimation of LV mass from LV linear dimensions on the basis of modeling the left ventricle as a prolate ellipse of revolution: LV mass $(\mathrm{g})=$ $0.8\left[1.04(\text { LVIDD + IVST + PWT })^{3}-\right.$ LVIDD $\left.^{3}\right]+0.6$, where LVIDD is LV internal end-diastolic dimension, IVST is end-diastolic interventricular septal wall thickness, and PWT is end-diastolic LV posterior wall thickness. $^{21}$

LV mass was indexed to BSA. LV mass was also indexed to height and to height ${ }^{2.7}$.

\section{Cutoff Limits for LV Hypertrophy}

The ASE/EAE guidelines suggest the following cutoffs for LV hypertrophy: LV mass/BSA > $95 \mathrm{~g} / \mathrm{m}^{2}$ (LV mass/height $>99 \mathrm{~g} / \mathrm{m}$, LV mass/ height $^{2.7}>44 \mathrm{~g} / \mathrm{m}^{2.7}$ ) for women and LV mass $/$ BSA $>115 \mathrm{~g} / \mathrm{m}^{2}$ (LV mass/height $>126 \mathrm{~g} / \mathrm{m}, \mathrm{LV}$ mass $/$ height $^{2.7}>48 \mathrm{~g} / \mathrm{m}^{2.7}$ ) for men. Values for mild LV hypertrophy are LV mass/BSA of 96 to $108 \mathrm{~g} / \mathrm{m}^{2}$ (LV mass/height, 100-115 g/m; LV mass/height ${ }^{2.7}$, 45$51 \mathrm{~g} / \mathrm{m}^{2.7}$ ) for women and LV mass/BSA of 116 to $131 \mathrm{~g} / \mathrm{m}^{2}$ (LV mass/height, $127-144 \mathrm{~g} / \mathrm{m}$; LV mass/height ${ }^{2.7}, 49-55 \mathrm{~g} / \mathrm{m}^{2.7}$ ) for men; values for moderate LV hypertrophy are LV mass/BSA of 109 to $121 \mathrm{~g} / \mathrm{m}^{2}$ (LV mass/height, $116-128 \mathrm{~g} / \mathrm{m}$; LV mass/height ${ }^{2.7}, 52-$ $58 \mathrm{~g} / \mathrm{m}^{2.7}$ ) for women and LV mass/BSA of 132 to $148 \mathrm{~g} / \mathrm{m}^{2}$ (LV mass/height, $145-162 \mathrm{~g} / \mathrm{m}$; LV mass/height ${ }^{2.7}, 56-63 \mathrm{~g} / \mathrm{m}^{2.7}$ ) for men; values for severe LV hypertrophy are LV mass/BSA $\geq 122 \mathrm{~g} / \mathrm{m}^{2}$ for women (LV mass/height $\geq 129 \mathrm{~g} / \mathrm{m}$, LV mass/ height $^{2.7} \geq 59 \mathrm{~g} / \mathrm{m}^{2.7}$ ) and LV mass $/$ BSA $\geq 149 \mathrm{~g} / \mathrm{m}^{2}$ (LV mass/height $\geq 163 \mathrm{~g} / \mathrm{m}$, LV mass $/$ height $^{2.7} \geq 64 \mathrm{~g} / \mathrm{m}^{2.7}$ ) for men. ${ }^{10}$

\section{Geometry}

Relative wall thickness (RWT) was measured using the formula $(2 \times$ PWT)/LVIDD, which permitted the categorization of an increase in LV mass as either concentric (RWT $\geq 0.42$ ) or eccentric (RWT < 0.42) hypertrophy ${ }^{10}$ and allowed the identification of concentric remodeling (normal LV mass with increased RWT).

\section{Clinical Data}

Age, sex, height, weight, BSA, body mass index, and cardiac rhythm were recorded at the time of echocardiography. History of 
cardiovascular diseases (prior acute coronary syndromes, including ST-segment elevation myocardial infarction and unstable angina or non-ST-segment elevation myocardial infarction, history of chronic coronary artery disease, prior acute or chronic heart failure, and prior stroke) was obtained using the hospital discharge codes of the public hospitals of Modena province. Risk factors were obtained retrospectively by manual review of electronic clinical notes of the public hospitals of the province. These electronic records allow assessment of outpatient visits as well as hospital discharge notes. To collect information on medications, we combined two methods: (1) we retrospectively reviewed through the electronic databases of Modena province public hospital discharge letters and ambulatory cardiology visits, and (2) we used the Emilia Romagna regional pharmacy centralized electronic database. This electronic database contains al prescriptions that are filled in all pharmacies of the region by all residents, regardless of the physician prescribing the medication. We examined all prescriptions filled from 60 days before to 30 days after the date of the exam

\section{Follow-Up}

The main end point was all-cause death. The secondary end point was cardiovascular morbidity, measured as the cumulative incidence of hospitalizations for cardiovascular disease. Included were hospitalizations for coronary disease, heart failure, ventricular arrhythmias, atrial fibrillation, lower limb critical ischemia, and stroke or transient ischemic attack. The diagnoses were derived from hospital discharge codes. In most cases, more than one code was reported, but only the first was considered to classify the cause of hospitalizations.

Follow-up information for death was obtained from the national death index, in which the status of all citizens is constantly updated and is $100 \%$ complete. In Italy, it is mandatory by law that all deceased patients be immediately registered in this national data bank Cardiovascular morbidity requiring hospitalization was assessed using the electronic archives of the health service of Modena province. All public hospitalization records of citizens resident in Modena province are stored in a digital format and may be accessed online after obtaining permission and an access password. This archive allows nearly complete knowledge of all clinical events requiring hospitalization in Modena province since 1999. Each electronic record includes up to 30 to 50 codes reflecting various diagnoses, complications, and procedures performed while patients were hospitalized. The diagnoses were classified according to the International Classification of Disease, Ninth Revision, Clinical Modification. We obtained computerized records of all incident hospitalizations for cardiovascular reasons as the principal diagnosis after the index echocardiographic study. In case of uncertainty in adjudicating events from this electronic database, the general practitioners were contacted by telephone. To ensure completeness of follow-up, only residents in Modena province were included in the study.

\section{Statistical Analysis}

Data are shown as percentages for categorical variables and as mean $\pm \mathrm{SD}$ for continuous variables. Comparisons across groups were made using $\chi^{2}$ tests for categorical variables and analysis of variance for continuous variables or Kruskal-Wallis tests for highly skewed variables.

Kaplan-Meier curves were constructed to show survival and the cumulative incidence of hospitalizations for cardiovascular diseases between patients with and without LV hypertrophy and across the
LV mass partition values. Event rates $\pm 1 \mathrm{SE}$ were estimated according to the Kaplan-Meier method, and groups were compared using a two-sided log-rank test for trend across the categories of LV mass.

Univariate and multivariate Cox regression analyses were used to estimate the relative risk for death and cardiovascular morbidity; these relative risks are presented as hazard ratios (HRs) with 95\% confidence intervals (CIs). Patients were censored at the end of their event free follow-up. Only the first incident event was included for each patient.

Patients were censored at the time of their first events. LV mass index was analyzed in several ways. First, the HR, was assessed using LV mass as a continuous variable; then, the HRs for patients with LV hypertrophy were compared with those of patients without hypertrophy; finally, to assess the prognostic effect of the ASE/EAE proposed classification scheme, the risk for adverse clinical outcomes was assessed among subjects in the four sex-specific proposed categories of indexed LV mass. For this purpose, we investigated whether the risk for adverse events differed among ASE/EAE categories using different multivariate statistical models: multiple-category models, in which the risk for adverse outcome in each category was compared with that associated with normal indexed LV mass, which served as the reference group (HR, 1), and trend models, in which we investigated whether there was a stepwise increase in the risk for adverse outcomes from one category to the next higher one.

Multivariate analyses included the following covariates: age (years), gender, atrial fibrillation, LV ejection fraction, WMSI, and significant valvular disease. Ancillary analyses were performed in the subgroup of patients in whom information on risk factors was available, and these covariates were added to the multivariate model. Finally, we tested for interactions between gender and LV hypertrophy grade and between cardiac rhythm and LV hypertrophy grade.

All tests were two tailed. $P$ values $<.05$ were considered statistically significant. All analyses were performed using SPSS version 15.0 for Windows (SPSS, Inc., Chicago, IL).

\section{RESULTS}

During the study period, 2,545 subjects (mean age, $61.9 \pm 15.8$ years $56.3 \%$ women) met the inclusion criteria and were considered for the analysis. The mean LV mass indexed to BSA was $107.5 \pm 37.3 \mathrm{~g} / \mathrm{m}^{2}$ among all subjects enrolled. According to the ASE/EAE guideline cutoffs for LV mass/BSA, 1,198 patients (47.1\%) were classified as having LV hypertrophy, and 1,347 (52.9\%) had normal LV mass indexes. LV hypertrophy was mild in 391 patients $(15.4 \%)$, moderate in 307 $(12.1 \%)$, and severe in 500 (19.6\%). Patients with higher categories of indexed LV mass were older, had higher body mass indexes, and were more likely to be women and to have hypertension, diabetes mellitus, hyperlipidemia, valvular heart disease, and atrial fibrillation, while there was no difference in smoking status. Higher degrees of LV hypertrophy were associated with worse systolic function and larger left atria (Table 1).

\section{Follow-Up Data}

Mortality. After a mean follow-up period of $2.5 \pm 1.2$ years, death occurred in 121 patients (4.8\%). Greater LV mass was associated with a linear increase in the risk for death (for each $1 \mathrm{~g} / \mathrm{m}^{2}$ increase in LV mass index, there was a $1.2 \%$ increase in the risk for death $(\mathrm{HR}, 1.012$ 95\% CI, 1.01-1.015; $P<.0001)$, and this association remained 
Table 1 Baseline clinical and echocardiographic characteristics according to LV hypertrophy severity

\begin{tabular}{|c|c|c|c|c|c|c|}
\hline Variable & $\begin{array}{c}\text { Total } \\
(n=2,545)\end{array}$ & $\begin{array}{c}\text { No LVH } \\
(n=1,347)\end{array}$ & $\begin{array}{l}\text { Mild LVH } \\
(n=391)\end{array}$ & $\begin{array}{l}\text { Moderate LVH } \\
\quad(n=307)\end{array}$ & $\begin{array}{l}\text { Severe LVH } \\
(n=500)\end{array}$ & $P$ for trend \\
\hline \multicolumn{7}{|l|}{ Baseline clinical characteristics } \\
\hline Age (y) & $61.9 \pm 15.8$ & $67.6 \pm 16.5$ & $64.9 \pm 14.3$ & $66.3 \pm 13.0$ & $68.6 \pm 12.9$ & $<.001$ \\
\hline Women & $1434(56.3 \%)$ & 725 (53.8\%) & 209 (53.5\%) & $176(57.3 \%)$ & $324(64.8 \%)$ & $<.001$ \\
\hline $\operatorname{BSA}\left(\mathrm{m}^{2}\right)$ & $1.82 \pm 0.22$ & $1.83 \pm 0.22$ & $1.82 \pm 0.21$ & $1.83 \pm 0.22$ & $1.78 \pm 0.20$ & $<.001$ \\
\hline $\mathrm{BMI}\left(\mathrm{m} / \mathrm{kg}^{2}\right)$ & $26.2 \pm 4.6$ & $25.4 \pm 4.6$ & $27.1 \pm 4.4$ & $26.6 \pm 4.3$ & $27.2 \pm 4.6$ & $<.001$ \\
\hline Hypertension $(n=1,851)$ & $1325(71.6 \%)$ & $553(60.2 \%)$ & $236(80.0 \%)$ & $193(80.8 \%)$ & $343(86.2 \%)$ & $<.001$ \\
\hline Diabetes mellitus $(n=1,851)$ & $228(12.3 \%)$ & $92(10.0 \%)$ & $36(12.2 \%)$ & 37 (15.5\%) & $63(15.8 \%)$ & .001 \\
\hline Hyperlipidemia $(n=1,850)$ & $444(24.0 \%)$ & $194(21.1 \%)$ & $77(26.1 \%)$ & $60(25.1 \%)$ & $113(28.4 \%)$ & .004 \\
\hline Smoking status $(n=1,851)$ & $187(10.1 \%)$ & $100(10.9 \%)$ & $29(9.8 \%)$ & $23(9.6 \%)$ & 35 (8.8\%) & .154 \\
\hline Prior acute coronary syndromes & $230(9.0 \%)$ & $98(7.3 \%)$ & $42(10.7 \%)$ & $27(8.8 \%)$ & $63(12.6 \%)$ & .001 \\
\hline History of chronic/stable coronary artery disease & $309(12.1 \%)$ & $125(9.3 \%)$ & $46(11.8 \%)$ & $51(16.6 \%)$ & $87(17.4 \%)$ & $<.001$ \\
\hline History of heart failure & $208(8.2 \%)$ & $75(5.6 \%)$ & $35(9.0 \%)$ & $35(11.4 \%)$ & $63(12.6 \%)$ & $<.001$ \\
\hline Prior stroke & $42(1.7 \%)$ & $17(1.3 \%)$ & $3(0.8 \%)$ & $7(2.3 \%)$ & $15(3.0 \%)$ & .007 \\
\hline Significant valvular heart disease & $274(10.8 \%)$ & $72(5.3 \%)$ & $42(10.7 \%)$ & $44(14.3 \%)$ & $116(23.2 \%)$ & $<.001$ \\
\hline Atrial fibrillation & $196(7.7 \%)$ & $76(5.6 \%)$ & $32(8.2 \%)$ & $27(8.8 \%)$ & $61(12.2 \%)$ & $<.001$ \\
\hline \multicolumn{7}{|l|}{ Echocardiographic characteristics } \\
\hline $\operatorname{LVEF}(\%)$ & $64.6 \pm 12.2$ & $66.1 \pm 10.5$ & $64.0 \pm 12.0$ & $64.0 \pm 12.7$ & $61.6 \pm 15.2$ & $<.001$ \\
\hline WMSI & $1.07 \pm 0.24$ & $1.03 \pm 0.14$ & $1.06 \pm 0.21$ & $1.09 \pm 0.28$ & $1.18 \pm 0.39$ & $<.001$ \\
\hline LAVI $\left(m L / m^{2}\right)(n=1,624)$ & $34.7 \pm 17.0$ & $29.4 \pm 13.5$ & $36.0 \pm 16.7$ & $36.2 \pm 15.7$ & $46.7 \pm 19.7$ & $<.001$ \\
\hline Normal diastolic function & $545(21.4 \%)$ & $366(27.2 \%)$ & $75(19.2 \%)$ & $49(16.0 \%)$ & $55(11.0 \%)$ & $<.001$ \\
\hline Grade I diastolic dysfunction & $411(16.1 \%)$ & $155(11.5 \%)$ & 77 (19.7\%) & $70(22.8 \%)$ & $109(21.8 \%)$ & $<.001$ \\
\hline Grade II diastolic dysfunction & $504(19.8 \%)$ & $233(17.3 \%)$ & $94(24.0 \%)$ & $64(20.8 \%)$ & $113(22.6 \%)$ & $<.001$ \\
\hline Grade III or IV diastolic dysfunction & $32(1.3 \%)$ & $11(0.8 \%)$ & $3(0.8 \%)$ & $7(2.3 \%)$ & $11(2.2 \%)$ & $<.001$ \\
\hline Undetermined diastolic dysfunction & $1053(41.4 \%)$ & $582(43.2 \%)$ & $142(36.3 \%)$ & $117(38.1 \%)$ & $212(42.4 \%)$ & $<.001$ \\
\hline $\mathrm{RWT}>0.42$ & 1007 (39.6\%) & $321(23.8 \%)$ & $193(49.4 \%)$ & $169(55.0 \%)$ & $324(64.8 \%)$ & $<.001$ \\
\hline Medications & $440(17.3 \%)$ & $231(17.1 \%)$ & $69(17.6 \%)$ & $50(16.3 \%)$ & $90(18.0 \%)$ & .796 \\
\hline Antiplatelet agents & $469(18.4 \%)$ & $250(18.6 \%)$ & $75(19.2 \%)$ & $48(15.6 \%)$ & $96(19.2 \%)$ & .911 \\
\hline$\beta$-blockers & $333(13.1 \%)$ & $156(11.6 \%)$ & $54(13.8 \%)$ & $37(12.1 \%)$ & $86(17.2 \%)$ & .004 \\
\hline Diuretics & $262(10.3 \%)$ & $140(10.4 \%)$ & $35(9.0 \%)$ & $34(11.1 \%)$ & $53(10.6 \%)$ & .823 \\
\hline Calcium channel blockers & $741(29.1 \%)$ & $379(28.1 \%)$ & $114(29.2 \%)$ & $91(29.6 \%)$ & $157(31.4 \%)$ & .170 \\
\hline ACE inhibitors/ARBs & $327(12.8 \%)$ & $174(12.9 \%)$ & $52(13.3 \%)$ & $41(13.4 \%)$ & $60(12.0 \%)$ & .698 \\
\hline Statins & $2(0.1 \%)$ & $1(0.1 \%)$ & 0 & 0 & $1(0.2 \%)$ & .543 \\
\hline Antiarrhythmic agents (class IA, IB) & $26(1.0 \%)$ & $17(1.3 \%)$ & $3(0.8 \%)$ & $2(0.7 \%)$ & $4(0.8 \%)$ & .278 \\
\hline Antiarrhythmic agents (class IC) & $18(0.7 \%)$ & $11(0.8 \%)$ & $2(0.5 \%)$ & $2(0.7 \%)$ & $3(0.6 \%)$ & .592 \\
\hline Amiodarone & $117(4.6 \%)$ & $60(4.5 \%)$ & $20(5.1 \%)$ & $13(4.2 \%)$ & $24(4.8 \%)$ & .823 \\
\hline Insulin/oral hypoglycemic drugs & $174(6.8 \%)$ & 87 (6.5\%) & $21(5.4 \%)$ & $18(5.9 \%)$ & $48(9.6 \%)$ & .051 \\
\hline Anticoagulants & $440(17.3 \%)$ & $231(17.1 \%)$ & $69(17.6 \%)$ & $50(16.3 \%)$ & $90(18.0 \%)$ & .796 \\
\hline
\end{tabular}

$A C E$, Angiotensin-converting enzyme; $A R B$, angiotensin receptor blocker; $B M I$, body mass index; $L A V I$, left atrial volume index; $L V H$, LV hypertrophy; $L V E F$, LV ejection fraction.

Data are expressed as mean \pm SD or as number (percentage). Unless otherwise specified, data were obtained for all 2,545 patients.

significant on multivariate analysis adjusting for age, gender, atrial fibrillation, LV ejection fraction, WMSI, and significant valvular disease (adjusted HR, 1.007; 95\% CI, 1.003-1.011; $P<.0001$ ). Patients with LV hypertrophy had significantly worse survival. Throughout the follow-up period, the presence of LV hypertrophy conferred a three fold increased risk for death (HR, 3.1; 95\% CI, 2.07-4.65; $P<.0001$ ), which remained significant after multivariate adjustment for age, gender, atrial fibrillation, LV ejection fraction, WMSI, and significant valvular disease (adjusted HR, 2.14; 95\% CI, 1.39-3.29; $P=.001$ ) There was a strong graded association between the severity of LV hypertrophy and survival $(P<.0001$; Figure 1$)$. At 3 years, Kaplan-Maier estimated survival was $97.4 \pm 0.5 \%$ in patients with normal LV mass and $94.3 \pm 1.5 \%$ in those with mild, $91.5 \pm 2.0 \%$ in those with moderate, and $90.9 \pm 1.5 \%$ in those with severe LV hypertrophy. Compared with patients with normal LV mass, the risk for death was greater than twofold in patients with mild LV hypertrophy (HR 2.17; 95\% CI, 1.23-3.81; $P=.007)$, greater than threefold in those with moderate LV hypertrophy (HR, 3.04; 95\% CI, 1.76-5.23; $P<$ .0001 ), and almost fourfold increased in those with severe LV hypertrophy (HR, 3.81 95\% CI, 2.43-5.97; $P<.0001$ ) (Table 2). After adjusting for age, gender, atrial fibrillation, LV ejection fraction, WMSI, and significant valvular disease, the strong association between LV hypertrophy severity grade and death remained significant (for mild LV hypertrophy: adjusted HR, 1.81; 95\% CI, 1.03-3.20; $P=.040$; for moderate LV hypertrophy: adjusted HR, 2.31; $95 \% \mathrm{CI}, 1.33-$ $4.01 ; P=.003$; and for severe LV hypertrophy: adjusted HR, 2.30; 95\% CI, 1.39-3.79; $P<.0001$ compared with normal LV mass) (Table 2). When RWT was added to this multivariate model, the results were similar (for mild LV hypertrophy: adjusted HR, 1.63; 95\% CI, 0.91-2.91; $P=.099$; for moderate LV hypertrophy: adjusted 


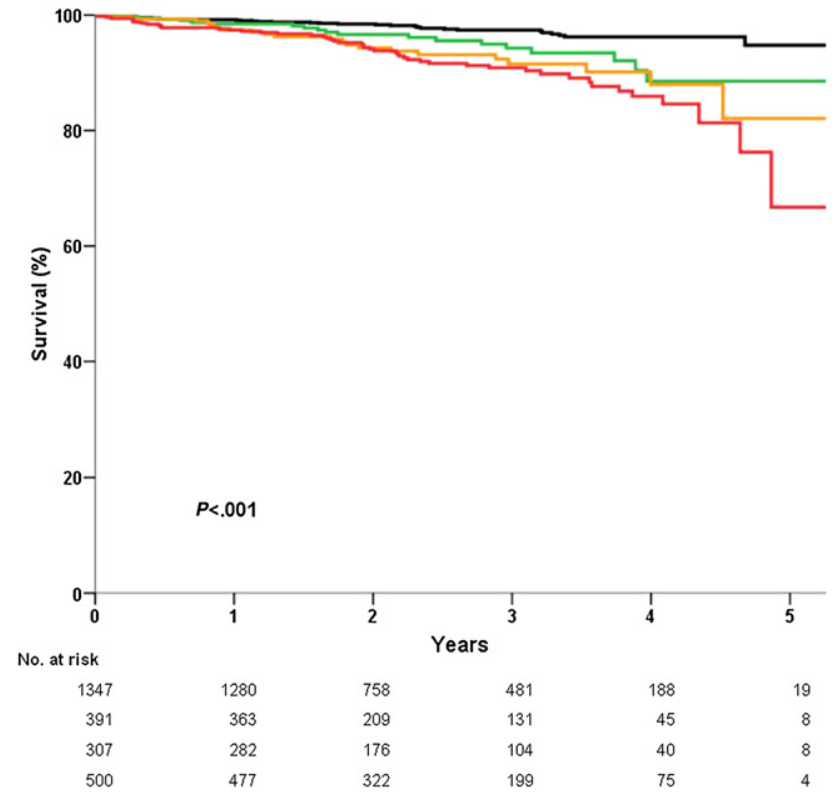

Figure 1 Overall mortality according to the ASE/EAE proposed classification scheme. The black line indicates patients without LV hypertrophy, the green line those with mild hypertrophy, the orange line those with moderate hypertrophy, and the red line those with severe hypertrophy.

HR, $2.01 ; 95 \% \mathrm{CI}, 1.13-3.57 ; P=.017$; and for severe LV hypertrophy: adjusted HR, 1.92; 95\% CI, 1.13-3.28; $P=.016$ compared with normal LV mass).

After further adjustment for history of acute coronary syndromes, history of chronic coronary artery disease, history of heart failure, and prior stroke, the association between LV mass partition values and death remained significant (for mild LV hypertrophy: HR, 1.73; 95\% CI, 0.98-3.06; $P=.059$; for moderate LV hypertrophy: HR, 2.16; 95\% CI, 1.24-3.76; $P=.007$; and for severe LV hypertrophy: HR, 2.15; 95\% CI, 1.31-3.54; $P=.003$ ). After further adjustment for cardiovascular risk factors (hypertension, diabetes mellitus, hyperlipidemia, and smoking) the association between LV hypertrophy severity and overall death persisted (for mild LV hypertrophy: adjusted HR, 1.77 ; 95\% CI, 0.91-3.46; $P=.092$; for moderate LV hypertrophy: adjusted HR, 2.47; 95\% CI, 1.31-4.67; $P=.005$; and for severe LV hypertrophy: adjusted HR, 2.15; 95\% CI, 1.19-3.89; $P=.011$ compared to normal). Furthermore, after adjusting for medications, the results were similar (data not shown). In the subgroup of patients in whom diastolic function was measurable, this variable was added to the multivariate model, and LV mass remained associated with death

Using trend models, there was a 50\% increase in hazard for death per increase in category of LV hypertrophy (HR, 1.54; 95\% CI, 1.341.77; $P<.0001$ ). On multivariate analysis, the adjusted HR was 1.31 (95\% CI, 1.12-1.53; $P=.001)$.

Cardiovascular Morbidity. During the follow-up period, 295 patients $(11.6 \%)$ were hospitalized for cardiovascular disease. Indexed LV mass was associated with an increased risk for cardiovascular hospitalization; for each $1 \mathrm{~g} / \mathrm{m}^{2}$ increase in LV mass index, the HR was 1.010 (95\% CI, 1.008-1.013; $P<.0001)$, and this association persisted on multivariate analysis adjusting for age, gender, atrial fibrillation, LVejection fraction, WMSI, and significant valvular disease (adjusted HR, 1.006; 95\% CI, 1.003-1.009; $P=.001$ ). Accordingly, the risk for hospitalization for cardiovascular diseases was significantly higher in patients with LV hypertrophy (HR, 2.58; 95\% CI, 2.02-3.31; $P<.001)$ compared with those with normal LV mass. This association remained significant after multivariate adjustment for age, gender, atrial fibrillation, LV ejection fraction, WMSI, and significant valvular disease (adjusted HR, 1.88; 95\% CI, 1.44-2.45; $P<.0001)$.

There was a significant risk gradient for cardiovascular morbidity across the degrees of LV hypertrophy (Figure 2). At 3 years, the Kaplan-Maier estimated cumulative incidence of hospitalizations for cardiovascular disease was $7.7 \%$ in patients with normal, $11.0 \%$ in those with mildly abnormal, $18.1 \%$ in those with moderately abnormal, and $24.2 \%$ in those with severely abnormal LV mass $(P<$ .0001). Table 3 shows the reasons for the first hospital admission: patients with more severe LV hypertrophy were more likely to be admitted for heart failure and atrial fibrillation but also ST-segment elevation myocardial infarction and lower limb critical ischemia. In multiple-category models, patients with greater LV mass had a higher incidence of hospitalization for cardiovascular diseases (compared with the reference group with normal LV mass) (for mild LV hypertrophy: HR, 1.47; 95\% CI, 1.00-2.15; $P=.048$; for moderate LV hypertrophy: HR, 2.50; 95\% CI, 1.77-3.54; $P<.001$; and for severe LV hypertrophy: HR, 3.48; 95\% CI, 2.65-4.59; $P<.0001$ ) (Table 2).

On multivariate analysis, after adjustment for age, gender, atrial fibrillation, LV ejection fraction, WMSI, and significant valvular disease, this graded association between LV mass category and cumulative incidence of cardiovascular hospitalizations remained strong (for mild LV hypertrophy: adjusted HR, 1.24; 95\% CI, 0.84-1.82; $P=.277$; for moderate LV hypertrophy: adjusted HR, 2.02; 95\% CI, $1.42-$ 2.88; $P<.0001$; and for severe LV hypertrophy: adjusted HR 2.38; 95\% CI, 1.75-3.23, $P<.0001$ ) (Table 2). When RWT was added to this multivariate model, the results were similar (for mild LV hypertrophy: adjusted HR, 1.28; 95\% CI, 0.84-1.93; $P=.24$; for moderate LV hypertrophy: adjusted HR, 1.77; 95\% CI, 1.172.67, $P=.007$; and for severe LV hypertrophy: adjusted HR, 2.28; 95\% CI, 1.59-3.27, $P<.001$ compared with normal LV mass). After further adjustment for history of acute coronary syndromes, history of chronic coronary artery disease, history of heart failure, and prior stroke, the association between LV mass partition values and cardiovascular morbidity remained significant (for mild LV hypertrophy: HR, 1.23 ; $95 \% \mathrm{CI}, 0.82-1.85, P=.323$; for moderate LV hypertrophy: HR, 1.60; 95\% CI, 1.08-2.39; $P=.021$; and for severe LV hypertrophy: HR, 2.07; 95\% CI, 1.48-2.88; $P<.001)$. After adding the risk factors to the multivariate model, the graded association persisted (for mildly abnormal LV mass: adjusted HR, 0.98; 95\% CI, 0.65-1.49; $P=$ .929; for moderately abnormal LV mass: adjusted $\mathrm{HR}, 1.73$; $95 \% \mathrm{CI}$, $1.20-2.50 ; P=.003$; and for severely abnormal LV mass: adjusted HR, $1.84 ; 95 \% \mathrm{CI}, 1.33-2.55 ; P<.0001$ ).

After adjusting for medications, the results were unchanged (data not shown)

Using trend models, there was a $43 \%$ increase in the hazard for hospitalization for cardiovascular disease per increase in category of LV hypertrophy (HR, 1.43; 95\% CI, 1.16-1.44, $P<.0001$ ). This association was maintained on multivariate analysis; the HR was 1.29 (95\% CI $1.12-1.53 ; P=.001)$ per increase in category of LV hypertrophy. In the subgroup of patients in whom diastolic function was measurable, this variable was added to the multivariate model, and LV mass maintained its association with cardiovascular morbidity.

Further testing for interaction for gender and cardiac rhythm was not significant (all $P$ values $>$.10). 
Table 2 Univariate and multivariate Cox regression analysis for death and cardiovascular hospitalizations

\begin{tabular}{|c|c|c|c|c|c|c|}
\hline Variable & Unadjusted HR & $95 \% \mathrm{Cl}$ & $P$ & Adjusted $\mathrm{HR}^{\star}$ & $95 \% \mathrm{Cl}$ & $P$ \\
\hline \multicolumn{7}{|l|}{ Death } \\
\hline \multicolumn{7}{|l|}{ Mass/BSA } \\
\hline & 1 & & 1 & & & \\
\hline Mild & 2.17 & $1.23-3.81$ & .007 & 1.81 & $1.03-3.20$ & .041 \\
\hline Moderate & 3.04 & $1.76-5.23$ & $<.001$ & 2.31 & $1.33-4.01$ & .003 \\
\hline Severe & 3.81 & $2.43-5.97$ & $<.001$ & 2.30 & $1.39-3.79$ & .001 \\
\hline \multicolumn{7}{|l|}{ Mass/height } \\
\hline Normal & 1 & & & 1 & & \\
\hline Mild & 1.45 & $0.83-2.54$ & .187 & 1.18 & $0.67-2.07$ & .563 \\
\hline Moderate & 2.62 & $1.54-4.44$ & $<.001$ & 1.97 & $1.15-3.37$ & .013 \\
\hline Severe & 2.33 & $1.50-3.61$ & $<.001$ & 1.52 & $0.95-2.42$ & .079 \\
\hline \multicolumn{7}{|c|}{ Mass/height ${ }^{2.7}$} \\
\hline & 1 & & 1 & & & \\
\hline Mild & 1.95 & $1.12-3.38$ & .017 & 1.58 & $0.90-2.76$ & .110 \\
\hline Moderate & 2.25 & $1.30-3.91$ & .004 & 1.73 & $0.99-3.02$ & .056 \\
\hline Severe & 2.93 & $1.85-4.63$ & .000 & 1.88 & $1.16-3.05$ & .010 \\
\hline \multicolumn{7}{|c|}{ Cardiovascular hospitalizations } \\
\hline \multicolumn{7}{|c|}{ Mass/BSA } \\
\hline & 1 & & 1 & & & \\
\hline Mild & 1.47 & $1.00-2.15$ & .048 & 1.24 & $0.84-1.82$ & .277 \\
\hline Moderate & 2.50 & $1.77-3.54$ & $<.001$ & 2.02 & $1.42-2.88$ & .001 \\
\hline Severe & 3.48 & $2.65-4.59$ & $<.001$ & 2.38 & $1.75-3.22$ & $<.001$ \\
\hline \multicolumn{7}{|l|}{ Mass/height } \\
\hline Normal & 1 & & & 1 & & .003 \\
\hline Mild & 1.73 & $1.19-2.50$ & .004 & 1.49 & $1.02-2.16$ & .039 \\
\hline Moderate & 2.50 & $1.71-3.64$ & $<.001$ & 2.01 & $1.38-2.95$ & $<.001$ \\
\hline Severe & 2.12 & $1.54-2.90$ & $<.001$ & 1.52 & $1.09-2.12$ & .013 \\
\hline \multicolumn{7}{|c|}{ Mass/height ${ }^{2.7}$} \\
\hline & 1 & & 1 & & & \\
\hline Mild & 1.72 & $1.18-2.52$ & .005 & 1.53 & $1.04-2.25$ & .031 \\
\hline Moderate & 2.55 & $1.78-3.65$ & $<.001$ & 2.09 & $1.45-3.01$ & $<.001$ \\
\hline Severe & 2.15 & $1.55-2.98$ & $<.001$ & 1.50 & $1.06-2.11$ & .021 \\
\hline
\end{tabular}

*Multivariate adjustment for age, sex, atrial fibrillation, LV ejection fraction, WMSI, and greater than moderate valvular disease.

LV Mass Indexed to Height and to Height ${ }^{2.7}$. Among all study subjects, the mean LV mass indexed to height was $117.9 \pm 42.2 \mathrm{~g} / \mathrm{m}$, and the mean LV mass indexed to height ${ }^{2.7}$ was $50.1 \pm 18.5 \mathrm{~g} / \mathrm{m}^{2.7}$ Applying the ASE/EAE partition values ${ }^{10}$ for mass indexed to height, 1,231 patients $(48.4 \%)$ had normal LV mass, $429(16.9 \%)$ had mild LV hypertrophy, 295 (11.6\%) had moderate LV hypertrophy, and 590 (23.2\%) had severe LV hypertrophy. Applying the ASE/EAE partition values ${ }^{10}$ for LV mass indexed to height ${ }^{2.7}, 1,147$ patients (45.1\%) had normal LV mass, 442 (17.4\%) had mild LV hypertrophy, 367 (14.4\%) had moderate LV hypertrophy, and 589 (32.1\%) had severe LV hypertrophy. Similarly to LV mass/BSA, the partition values for LV mass/height and LV mass/height ${ }^{2.7}$ were associated with a significant and graded increased risk for death and cardiovascular hospitalizations; such associations remained significant on multivariate analyses adjusting for age, gender, atrial fibrillation, LV ejection fraction, WMSI, and significant valvular disease (Table 2).

\section{Geometry}

In our population, $40 \%$ of patients had normal geometry, $13 \%$ had concentric remodeling, 27\% had eccentric hypertrophy, and 20\% had concentric hypertrophy. LV concentric and eccentric hypertrophy were associated with death (HR, 3.47; 95\% CI, 2.14-5.62,
$P<.001$; and HR, 2.96; 95\% CI, 1.77-4.97; $P<.001)$ and cardiovascular hospitalizations (HR, 1.95; 95\% CI, 1.39-2.73; $P<.001$; and HR, 2.86; 95\% CI, 2.06-3.99; $P<.001$ ), while concentric remodeling was not (HR, 1.22; $95 \% \mathrm{CI}, 0.55$ to $2.71 ; P=.630$ for death; and HR, 1.24; 95\% CI, 0.76-2.02; $P=.385$ ). Results were similar on multivariate analysis (data not shown). Thus, the main determinant of prognosis was the presence of LV hypertrophy rather than the type of remodeling.

\section{DISCUSSION}

To date, three approaches have been used for the definition of cutoff values to categorize echocardiographic parameters ${ }^{10}:$ (1) the method most widely used is statistical, based on standard deviations above and below the reference limits (mean values) or on percentiles obtained from a group of apparently healthy individuals; (2) values categorized according to cardiovascular outcomes on follow-up, whereby limits are identified that indicate increasing risk with increasing grade of deviation; and (3) choice of cutoffs on the basis of expert opinion. To comply with the need to standardize echocardiographic measures, $^{22}$ in 2005 , the ASE and EAE proposed categories for the description of values exceeding the reference intervals. ${ }^{10}$ 


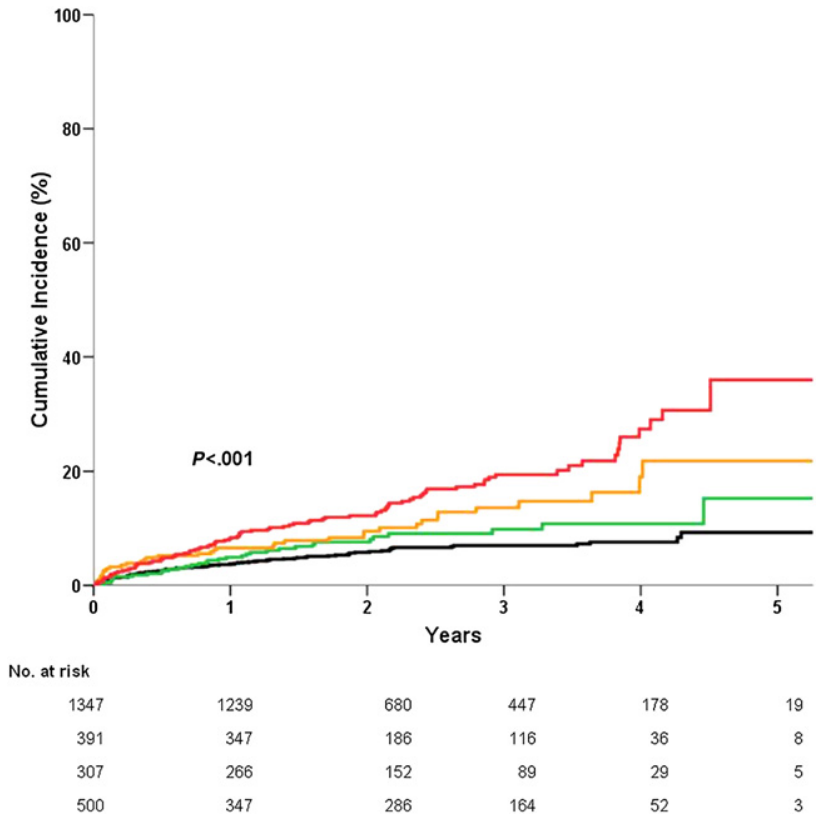

Figure 2 Cumulative incidence of hospitalizations for cardiovascular disease according to the ASE/EAE proposed classification scheme. The black line indicates patients without LV hypertrophy, the green line those with mild hypertrophy, the orange line those with moderate hypertrophy, and the red line those with severe hypertrophy.

Current reference values for linear LV dimensions, including the calculated LV mass using LV linear dimensions indexed to BSA, were obtained from the distribution in relation to reference limits in a ethnically heterogeneous North American population of 510 white African American, and American Indian adults (the first method). ${ }^{11}$ Recently, percentile-derived values from a large-scale, populationbased study in apparently healthy individuals as well as those with prevalent disease of 5,000 mid-Europeans revealed comparable cutoff values. ${ }^{23}$ Ideally, to provide normative data about the degree to which echocardiographic LV hypertrophy measurements deviate from normal, a practical approach that predicts outcomes or prognosis would be preferred. However, to the best of our knowledge, whether the ASE/EAE categorization of LV hypertrophy is able to predict prog nosis in clinical practice has never been evaluated. Therefore, the rationale of the current investigation was to provide the first systematic external documentation (clinical validation by application). The longitudinal design of the present study facilitated this type of validation We analyzed a broad sample of elective adult outpatients that included healthy and diseased individuals. Our data showed that echocardiographically detected LV mass and the partition values proposed by the ASE and EAE were strongly related to death and cardiovascular morbidity and were able to classify a risk gradient. The greater the grade of LV hypertrophy severity, the worse the prognosis. Results of trend models indicated an adjusted stepwise increase in hazard per category increase in LV hypertrophy of about 30\% for both death and cardiovascular morbidity. The incremental adverse impact associated with mild, moderate, and severe LV hypertrophy (compared with normal reference range) persisted in multivariate analyses adjusting for the effect of other known predictors and was generally consistent within the various statistical models explored.

LV hypertrophy is related to a vast number of identifiable conditions, including advanced age, hypertension, obesity, diabetes, prior myocardial infarction, and valvular heart disease. In addition, several other risk factors (unmeasured in our study), including sodium intake, neurohumoral influences, insulin, other growth factors and genetic risk factors, may be involved in modulating a complex trait such as the development and pattern of LV hypertrophy.

Although considerable interest exists in recognizing putative risk factors that modulate LV hypertrophy, the research in this topic remains scarce, with insufficient clinical data. Therefore, the mechanisms responsible for LV mass increase remain elusive and are beyond the scope of this study. ${ }^{24}$

We can speculate that increased LV mass is a marker of global cardiovascular risk burden because it reflects and integrates the long-term level of activity of factors inducing progression of atherosclerosis. $^{25}$ Beyond traditional risk factors, there may be others (unmeasured in our report), including biologic factors and hemodynamic (such as arterial stiffness) and nonhemodynamic (i.e., insulin and insulin growth factors) factors that are able to contemporaneously induce LV hypertrophy and progression or destabilization of atherosclerotic lesions. On the other hand, LV hypertrophy may represent a mediator of myocardial ischemia, with potential evolution toward arrhythmias and heart failure.

Our data confirmed ${ }^{26}$ that echocardiographic LV hypertrophy identified a population at high risk for cardiovascular disease. Subjects with more severe grades of LV hypertrophy were older and more likely to have hypertension and diabetes. These factors, associated with LV hypertrophy, are atherogenic. Therefore, LV mass may be considered as an integrated marker of atherosclerosis and absolute cardiovascular risk. ${ }^{23}$ Accordingly, the present study demonstrated a relationship between LV hypertrophy grade and incident hospitalizations for lower limb critical ischemia. Nevertheless, in our population, echocardiographic LV hypertrophy independently predicted an increased risk for death and cardiovascular morbidity after adjustment for other major risk factors. Therefore, increased LV mass may also represent a direct causative factor for events; in particular, LV hypertrophy may mediate myocardial ischemia, with potential evolution toward heart failure and arrhythmias. Notably, the present study, documenting a significant increased risk for hospitalizations for heart failure per category of LV mass, corroborates the hypothesis of LV hypertrophy as a mediator of cardiovascular events. Unfortunately, in clinical practice, it is generally impossible to establish a link between LV hypertrophy and one or more potential causative factors in individual patients. ${ }^{23}$

Analyses from the Framingham Heart Study ${ }^{27}$ and elsewhere $28-30$ have unequivocally demonstrated the prognostic value of echocardiographically detected LV hypertrophy as a continuous measure to predict cardiovascular disease events and survival. Our study confirmed these findings by showing that a greater indexed LV mass as a continuous variable was associated with an increasing risk for death and cardiovascular morbidity. ${ }^{1}$

Our study expands these findings to the categories of LV hypertrophy. In clinical practice, the measures obtained at the echocardiographic exam must be interpreted and qualified such that the exam is informative and useful for nonspecialists. ${ }^{22}$ To this end, the ASE/EAE classification was created to enhance the comprehensibility of measurements to clinicians and to promote uniformity in describing measurements. Our study validated such a categorization scheme in relation to long-term prognosis in a large group of unselected subjects referred for echocardiography. LV mass partition values were able to classify a risk gradient regardless of indexation to BSA, height, or height ${ }^{2.7}$.

Only the study of Vasan et al. ${ }^{2}$ subdivided on an ordinal scale the values exceeding reference limits for practical use by clinicians. 
Table 3 Causes of incident hospitalizations for cardiovascular disease

\begin{tabular}{|c|c|c|c|c|c|c|}
\hline Variable & Total & No LVH & Mild LVH & Moderate LVH & Severe LVH & $P$ for trend \\
\hline STEMI & $13(0.5 \%)$ & $4(0.3 \%)$ & $1(0.3 \%)$ & 0 & $8(1.6 \%)$ & .005 \\
\hline UA-NSTEMI & $38(1.5 \%)$ & $17(1.3 \%)$ & $9(2.3 \%)$ & $8(2.6 \%)$ & $4(0.8 \%)$ & .956 \\
\hline Stable angina & $15(0.6 \%)$ & $7(0.3 \%)$ & $3(0.8 \%)$ & $3(1.0 \%)$ & $2(0.4 \%)$ & .959 \\
\hline Coronary artery disease & $31(1.2 \%)$ & $12(0.9 \%)$ & $7(1.8 \%)$ & $6(2.0 \%)$ & $6(1.2 \%)$ & .328 \\
\hline Atrial fibrillation & $48(1.9 \%)$ & $20(1.5 \%)$ & $5(1.3 \%)$ & $10(3.3 \%)$ & $13(2.6 \%)$ & .042 \\
\hline Ventricular arrhythmias & $5(0.2 \%)$ & $2(0.1 \%)$ & $2(0.5 \%)$ & 0 & $1(0.2 \%)$ & .977 \\
\hline Heart failure & $80(3.1 \%)$ & $24(1.8 \%)$ & $13(3.3 \%)$ & $8(2.6 \%)$ & $35(7.0 \%)$ & $<.001$ \\
\hline Stroke/TIA & $26(1.0 \%)$ & $11(0.8 \%)$ & $4(1.0 \%)$ & $6(2.0 \%)$ & $5(1.0 \%)$ & .374 \\
\hline Lower limb critical ischemia & $41(1.6 \%)$ & $16(1.2 \%)$ & $3(0.8 \%)$ & $11(3.6 \%)$ & $11(2.2 \%)$ & .020 \\
\hline
\end{tabular}

LVH, LV hypertrophy; NSTEMI, non-ST-segment elevation myocardial infarction; STEMI, ST-segment elevation myocardial infarction; TIA, transient ischemic attack; $U A$, unstable angina.

These investigators examined the distribution of measurements in a broad sample $(n=4,957)$ and classified the measurements according to increasing deviation from the height-specific and sex-specific reference limits and the 95th, 98th, and 99th percentile values for the broad sample. To validate this categorization scheme, they used multivariate proportional-hazards regression to assess the relations of LV mass categories to risk for cardiovascular events and found a 1.3-fold adjusted risk for cardiovascular disease events per category of LV mass, which is almost identical to what we found in the present study using trend models.

\section{Limitations and Strengths}

The study population was referral based, and the extent to which the data can be generalized to other populations remains unknown. In particular, because the vast majority of subjects enrolled were Caucasian, results may not be applicable to other ethnicities.

Information on risk factors was retrievable for only $73 \%$ of the patients. For this reason, multivariate analysis including risk factors as covariates was performed separately as ancillary analysis.

The use of linear dimensions for LV mass determination presents potential limitations. Linear measurements are based on the assumption that the left ventricle is represented by a prolate ellipse, while cardiac disease may result in distorted LV geometry with the possibility of underestimating or overestimating LV mass. ${ }^{10}$ Furthermore, cubing linear dimensions within the formula can multiply even small errors. However, LV mass obtained with this method has been well validated by necropsy. ${ }^{21}$

It is possible for a patient to shift between categories simply on the basis of limitations in the reproducibility of echocardiographic measurements. ${ }^{31}$ However, linear dimensions can be obtained from either 2D-guided M-mode images or directly from correctly aligned 2D images with reasonable agreement ${ }^{32}$ and good reproducibility. ${ }^{33}$ Particularly, the latter method overcomes the common problem of oblique parasternal images that result in overestimation of cavity by M-mode imaging. ${ }^{31}$

We acknowledge that there are some limitations using hospital discharge International Classification of Disease, Ninth Revision, Clinical Modification codes as the basis for a definition of cardiovascular morbidity, because it does not equate to the stringent criteria used, for example, in the Framingham study, with a risk for misclassification. ${ }^{1}$ However, this setting provided an opportunity to collect follow-up information in $100 \%$ of the patients by passive surveillance through the province inpatient medical records. Therefore, despite difficulties in the interpretation of administrative hospital data, hospital admissions involving diagnoses of cardiovascular disease can provide valuable information about the incidence of cardiovascular complications in patients with LV hypertrophy.

Other three-dimensional techniques such as magnetic resonance imaging would have provided greater accuracy and reproducibility, but this is beyond the scope of the study, and B-mode echocardiography is at present the best method for screening large populations.

Finally, although we have demonstrated significant prognostic value of this categorization scheme, the therapeutic implications of this classification, if any, are unknown.

Strengths of the study include the real-time contemporary measurement of LV mass in a large number of unselected outpatients and completeness of follow-up.

\section{CONCLUSIONS}

In a large population of unselected outpatients, we demonstrated the ability of the classification scheme of LV hypertrophy proposed by the ASE and EAE to predict mortality and cardiovascular morbidity. This considerably enhances its utility for clinicians and may improve communication among laboratories.

\section{REFERENCES}

1. Levy D, Garrison RJ, Savage DD, Kannel WB, Castelli WP. Prognostic implications of echocardiographically determined left ventricular mass in the Framingham Heart Study. N Engl J Med 1990;322:1561-6.

2. Vasan RS, Larson MG, Levy D, Evans JC, Benjamin EJ. Distribution and categorization of echocardiographic measurements in relation to reference limits: the Framingham Heart Study: formulation of a height- and sex-specific classification and its prospective validation. Circulation 1997;96:1863-73.

3. Tsang TS, Barnes ME, Gersh BJ, Takemoto Y, Rosales AG, Bailey KR, et al. Prediction of risk for first age-related cardiovascular events in an elderly population: the incremental value of echocardiography. J Am Coll Cardiol 2003;42:1199-205

4. Gardin JM, McClelland R, Kitzman D, Lima JA, Bommer W, Klopfenstein HS, et al. M-mode echocardiographic predictors of six- to seven-year incidence of coronary heart disease, stroke, congestive heart failure, and mortality in an elderly cohort (the Cardiovascular Health Study). Am J Cardiol 2001;87:1051-7.

5. Bolognese L, Dellavesa P, Rossi L, Sarasso G, Bongo AS, Scianaro MC. Prognostic value of left ventricular mass in uncomplicated acute 
myocardial infarction and one-vessel coronary artery disease. Am J Cardiol 1994;73:1-5.

6. Cooper RS, Simmons BE, Castaner A, Santhanam V, Ghali J, Mar M. Left ventricular hypertrophy is associated with worse survival independent of ventricular function and number of coronary arteries severely narrowed. Am J Cardiol 1990;65:441-5.

7. Stevens SM, Farzaneh-Far R, Na B, Whooley MA, Schiller NB. Development of an echocardiographic risk-stratification index to predict heart failure in patients with stable coronary artery disease: the Heart and Soul study. JACC Cardiovasc Imaging 2009;2:11-20.

8. Verma A, Meris A, Skali H, Ghali JK, Arnold JM, Bourgoun M, et al. Prognostic implications of left ventricular mass and geometry following myocardial infarction: the VALIANT (Valsartan in Acute Myocardial Infarction) Echocardiographic Study. JACC Cardiovasc Imaging 2008; 1:582-91.

9. Quinones MA, Greenberg BH, Kopelen HA, Koilpillai C, Limacher MC, Shindler DM, et al. Echocardiographic predictors of clinical outcome in patients with left ventricular dysfunction enrolled in the SOLVD registry and trials: significance of left ventricular hypertrophy. J Am Coll Cardiol 2000; 35:1237-44.

10. Lang RM, Bierig M, Devereux RB, Flachskampf FA, Foster E, Pellikka PA, et al. Recommendations for chamber quantification: a report from the American Society of Echocardiography's Guidelines and Standards Committee and the Chamber Quantification Writing Group, developed in conjunction with the European Association of Echocardiography, a branch of the European Society of Cardiology. J Am Soc Echocardiogr 2005; 18: 1440-63.

11. Ilercil A, O'Grady MJ, Roman MJ, Paranicas M, Lee ET, Welty TK, et al. Reference values for echocardiographic measurements in urban and rural populations of differing ethnicity: the Strong Heart Study. J Am Soc Echocardiogr $2001 ; 14: 601-11$.

12. Beller GA, Bonow RO, Fuster V. ACCF 2008 Recommendations for Training in Adult Cardiovascular Medicine Core Cardiology Training (COCATS 3) (revision of the 2002 COCATS training statement). J Am Coll Cardiol 2008;51:335-8.

13. Quinones MA, Waggoner AD, Reduto LA, Nelson JG, Young JB, Winters WL Jr., et al. A new, simplified and accurate method for determining ejection fraction with two-dimensional echocardiography. Circulation 1981;64:744-53.

14. Amico AF, Lichtenberg GS, Reisner SA, Stone CK, Schwartz RG, Meltzer RS. Superiority of visual versus computerized echocardiographic estimation of radionuclide left ventricular ejection fraction. Am Heart J 1989;118:1259-65.

15. Bursi F, Weston SA, Redfield MM, Jacobsen SJ, Pakhomov S, Nkomo VT, et al. Systolic and diastolic heart failure in the community. JAMA 2006; 296:2209-16.

16. Redfield MM, Jacobsen SJ, Burnett JC Jr., Mahoney DW, Bailey KR, Rodeheffer RJ. Burden of systolic and diastolic ventricular dysfunction in the community: appreciating the scope of the heart failure epidemic. JAMA 2003;289:194-202.

17. Barbieri A, Bursi F, Zanasi V, Veronesi B, Cioni E, Modena MG. Left atrium reclassified: application of the American Society of Echocardiography/European Society of Cardiology cutoffs to unselected outpatients referred to the echocardiography laboratory. I Am Soc Echocardiogr 2008;21:433-8.

18. Ujino K, Barnes ME, Cha SS, Langins AP, Bailey KR, Seward JB, et al. Two-dimensional echocardiographic methods for assessment of left atrial volume. Am J Cardiol 2006;98:1185-8.
19. Ommen SR, Nishimura RA, Appleton CP, Miller FA, Oh JK, Redfield MM, et al. Clinical utility of Doppler echocardiography and tissue Doppler imaging in the estimation of left ventricular filling pressures: a comparative simultaneous Doppler-catheterization study. Circulation 2000;102: 1788-94.

20. Bonow RO, Carabello BA, Chatterjee K, de Leon AC Jr., Faxon DP, Freed MD, et al. ACC/AHA 2006 guidelines for the management of patients with valvular heart disease: a report of the American College of Cardiology/American Heart Association Task Force on Practice Guidelines (Writing Committee to Revise the 1998 Guidelines for the Management of Patients With Valvular Heart Disease) developed in collaboration with the Society of Cardiovascular Anesthesiologists endorsed by the Society for Cardiovascular Angiography and Interventions and the Society of Thoracic Surgeons. J Am Coll Cardiol 2006;48:e1-148.

21. Devereux RB, Alonso DR, Lutas EM, Gottlieb GJ, Campo E, Sachs I, et al. Echocardiographic assessment of left ventricular hypertrophy: comparison to necropsy findings. Am J Cardiol 1986;57:450-8.

22. Vasan RS, Levy D, Larson MG, Benjamin EJ. Interpretation of echocardiographic measurements: a call for standardization. Am Heart J 2000;139 412-22.

23. Wild PS, Sinning CR, Roth A, Wilde S, Schnabel RB, Lubos E, et al. Distribution and categorization of left ventricular measurements in the general population: results from the population-based Gutenberg Heart Study. Circ Cardiovasc Imaging; 3:604-13

24. Drazner MH. The progression of hypertensive heart disease. Circulation 2011;123:327-34

25. Verdecchia P, Angeli F, Achilli P, Castellani C, Broccatelli A, Gattobigio R, et al. Echocardiographic left ventricular hypertrophy in hypertension: marker for future events or mediator of events? Curr Opin Cardiol 2007;22:329-34.

26. Levy D, Murabito JM, Anderson KM, Christiansen JC, Castelli WP. Echocardiographic left ventricular hypertrophy: clinical characteristics. The Framingham Heart Study. Clin Exp Hypertens A 1992;14:85-97.

27. Levy D, Garrison RJ, Savage DD, Kannel WB, Castelli WP. Left ventricular mass and incidence of coronary heart disease in an elderly cohort. The Framingham Heart Study. Ann Intern Med 1989;110:101-7.

28. Schillaci G, Verdecchia P, Porcellati C, Cuccurullo O, Cosco C, Perticone F. Continuous relation between left ventricular mass and cardiovascular risk in essential hypertension. Hypertension 2000;35:580-6.

29. Koren MJ, Devereux RB, Casale PN, Savage DD, Laragh JH. Relation of left ventricular mass and geometry to morbidity and mortality in uncom plicated essential hypertension. Ann Intern Med 1991;114:345-52.

30. Drazner MH, Rame JE, Marino EK, Gottdiener JS, Kitzman DW, Gardin JM, et al. Increased left ventricular mass is a risk factor for the development of a depressed left ventricular ejection fraction within five years: the Cardiovascular Health Study. J Am Coll Cardiol 2004;43: 2207-15.

31. Gottdiener JS, Bednarz J, Devereux R, Gardin J, Klein A, Manning WJ, et al American Society of Echocardiography recommendations for use of echocardiography in clinical trials. J Am Soc Echocardiogr 2004;17:1086-119.

32. Myerson SG, Montgomery HE, World MJ, Pennell DJ. Left ventricular mass: reliability of M-mode and 2-dimensional echocardiographic formulas. Hypertension 2002;40:673-8.

33. Palmieri V, de Simone G, Roman MJ, Schwartz JE, Pickering TG Devereux RB. Ambulatory blood pressure and metabolic abnormalities in hypertensive subjects with inappropriately high left ventricular mass. Hypertension 1999;34:1032-40. 